\title{
Imaging Characteristics of Cerebrovascular Arteriopathy and Stroke in Hutchinson-Gilford Progeria Syndrome
}

\author{
V.M. Silvera, L.B. Gordon, D.B. Orbach, S.E. Campbell, J.T. Machan, and N.J. Ullrich
}

\begin{abstract}
BACKGROUND AND PURPOSE: HGPS is a rare disorder of segmental aging, with early morbidity from cardiovascular and cerebrovascular disease. The goal of this study was to identify the neurovascular features, infarct type, topography, and natural history of stroke in the only neurovascular imaging cohort study of HGPS.
\end{abstract}

MATERIALS AND METHODS: We studied 25 children with confirmed diagnoses of HGPS and neuroimaging studies available for review. Relevant clinical information was abstracted from medical records.

RESULTS: We identified features suggestive of a vasculopathy unique to HGPS, including distinctive intracranial steno-occlusive arterial lesions, basal cistern collateral vessels, and slow compensatory collateral flow over the cerebral convexities. The arterial pathology in the neck consisted of distal vertebral artery stenosis with prominent collateral vessel formation as well as stenosis and calcification of both the cervical internal and common carotid arteries. Radiographic evidence of infarction was found in $60 \%$ of patients, of which half were likely clinically silent. Both large- and small-vessel disease was observed, characterized by arterial territorial, white matter, lacunar, and watershed infarcts.

CONCLUSIONS: We report a unique intracranial and superior cervical arteriopathy in HGPS distinct from other vasculopathies of childhood, such as Moyamoya, and cerebrovascular disease of aging, including atherosclerosis. Arterial features of the mid and lower neck are less distinctive. For the first time, we identified early and clinically silent strokes as a prevalent disease characteristic in HGPS. Longitudinal analysis of stroke incidence and vasculopathy may provide an outcome measure for future treatment interventions for children with HGPS.

ABBREVIATIONS: $\mathrm{ACA}=$ anterior cerebral artery; $\mathrm{CCA}=$ common carotid artery; HGPS = Hutchinson-Gilford Progeria Syndrome; PCA = posterior cerebral artery; $\mathrm{VA}=$ vertebral artery

$\mathbf{H}$ GPS (Progeria) is a rare fatal syndrome of segmental premature aging that begins in early childhood, with an estimated overall incidence of 1 in 4-8 million. ${ }^{1}$ HGPS arises from a sporadic autosomal dominant mutation of the LMNA gene, which, in

Received July 2, 2012; accepted after revision July 20.

From the Departments of Radiology (V.M.S., D.B.O.), Anesthesia (L.B.G.), and Neurology (N.J.U.), Boston Children's Hospital, Harvard Medical School, Boston, Massachusetts; Department of Pediatrics (L.B.G.), Hasbro Children's Hospital, Providence, Rhode Island; Warren Alpert Medical School (L.B.G., J.T.M.) and Center for Gerontology and Healthcare Research (S.E.C.), Brown University, Providence, Rhode Island; and Departments of Biostatistics, (J.T.M.), Orthopaedics, Surgery, and Psychology (J.T.M.), Rhode Island Hospital, Providence, Rhode Island.

This work was funded by The Progeria Research Foundation (PRF2002-DB) to L.B.G and S.E.C.

Please address correspondence to Nicole J. Ullrich, MD, PhD, Department of Neurology, Boston Children's Hospital, 300 Longwood Ave, Boston, MA 02446; e-mail: Nicole.ullrich@childrens.harvard.edu

三 Indicates article with supplemental on-line tables

http://dx.doi.org/10.3174/ajnr.A3341 turn, produces an aberrant form of the inner nuclear membrane protein lamin A, called progerin. ${ }^{2,3}$ Progerin production results in an aberrant nuclear shape and the dysregulated expression of hundreds of genes. ${ }^{4}$ Progerin is expressed in differentiated cell types, including vascular smooth muscle cells, endothelial cells, and adventitial fibroblasts, ${ }^{5,6}$ all of which are integral to vascular structure and function. Additionally, progerin has been linked to the normal aging process in healthy individuals, ${ }^{7}$ in whom vascular progerin increases throughout life. Children with HGPS experience the early onset of many disease processes commonly observed in a normally aging population, including cardiovascular disease, coronary artery atherosclerosis, myocardial infarction, and stroke. As such, the role of progerin as it relates to aspects of cardiovascular and cerebrovascular pathology in HGPS and atherosclerosis in the aged is of interest. ${ }^{6}$

Children with HGPS experience early failure to thrive with progressive sclerodermatous skin changes, craniofacial disproportion, hair loss, and limb wasting. Cognitive function is normal barring stroke-induced deficits. ${ }^{8}$ 
Cardiovascular and cerebrovascular events in patients with HGPS result in significant morbidity and mortality, with an early death at a mean age of 13 years. ${ }^{1}$ The latter stage of HGPS is marked by episodes of angina, myocardial infarction, TIAs, and stroke. Cardiovascular disease in HGPS has been characterized in several prospective cohort studies. ${ }^{9,10}$ Postmortem studies have identified fatty plaques and complex calcified lesions within coronary arteries. ${ }^{6,11-13}$ Despite the prevalence of cerebrovascular disease in HGPS, little is known about the nature of strokes and vascular characteristics of the head and neck in these patients. Carotid sonography has identified atherosclerotic plaque in cervical arteries ${ }^{9,10}$ and isolated case reports of ICA and VA stenoses and occlusions in children with HGPS have presumed atherosclerosis as the underlying etiology in the absence of histologic confirmation. The goal of this study was to determine the incidence, imaging characteristics, and natural history of cerebrovascular disease in a large cohort of children with HGPS and to compare these findings with the established characteristics of cerebrovascular disease in the aging non-HGPS population.

\section{MATERIALS AND METHODS Patients and Data}

Patients were identified from The Progeria Research Foundation Medical and Research Database (Brown University Center for Gerontology and Health Care Research, Providence, Rhode Island). Eligible individuals had a genetic and/or clinical diagnosis determined by the phenotype of HGPS and at least 1 MRI or CT of the head or neck. Indications for neuroimaging and coincident neurologic assessments were abstracted from medical records. We have previously reported on the neuroanatomic features of this cohort; numbers pertaining to study indications in this report differ slightly due to various types of examinations reviewed. ${ }^{14}$

\section{Neuroimaging Studies}

A neuroradiologist (V.M.S.) and a neurologist (N.J.U.) interpreted all neuroimaging studies in consensus reading sessions. Neurovascular studies were also reviewed by a neuroradiologist/ neurointerventionalist (D.B.O.). There was considerable variability in imaging available from different institutions; therefore, the frequency of findings was expressed as the number of patients with a positive finding relative to the number of patients in whom imaging allowed adequate assessment of that finding, rather than as a proportion of the entire cohort, so as not to under-represent the prevalence of the finding in the population as a whole. The degree of cerebral and cervical arterial stenoses was assessed visually. High-grade stenosis resulting in complete occlusion or vascular amputation without residual signal flow downstream was termed "occlusion", whereas arterial stenosis resulting in a decrease in the vascular diameter with residual flow signal downstream was termed "narrowing". Mild stenosis assessed at $<25 \%$ was not considered narrowed. Arterial enlargement was defined as visualization of an artery not normally visualized and/or visualization of an artery that was increased in luminal diameter. Limitations of the range and quality of imaging studies in the cohort precluded more detailed grading of stenosis and compensatory enlargement. Regardless of laterality or side, infarct type and dis- tribution and arterial stenoses were counted as a maximum of 1 per patient.

\section{Statistical Analysis}

Descriptive statistics were used to assess the prevalence of vascular events and vascular anatomic features. If a stroke was present on the first available imaging study, whether acute or chronic, the patient's age at that scan time was taken as the age of first identification of a stroke. A Kaplan-Meier survival analysis was then performed to estimate the stroke-free probability.

\section{Institutional Approvals}

This Health Insurance Portability and Accountability Act-compliant study was approved by the institutional review boards of Rhode Island Hospital and Brown University. Informed consent was obtained by the principal investigator (L.B.G.).

\section{RESULTS}

\section{Patient Demographics}

On-line Table 1 lists individual patient characteristics and types of neuroimaging studies included in this study. Twentyfive children (11 females, 14 males) between birth and 14.1 years (mean age, 6.5 years) were studied. Mutational analysis revealed 18 children with classic heterozygous c.1824 C>T (G608G) mutations, 2 children with nonclassic heterozygous c.1822 G>A (G608S) mutations, and 1 with nonclassic c. 1968 $+1 \mathrm{G}>\mathrm{A} .{ }^{15}$ On the basis of phenotype, 3 children were presumed to have a classic mutation and 1 was presumed to have a nonclassic progerin-producing mutation.

\section{Neuroimaging Characteristics}

Twenty-five children provided 126 imaging studies from birth to 14.1 years (On-line Table 1). Of these, 19 patients had longitudinal follow-up imaging with a mean of 4.1 scans/patient and a mean duration of imaging follow-up of 2.8 years. The most frequent indications for imaging were evaluation of acute neurologic symptoms, diagnostic evaluation, or follow-up of prior abnormal imaging findings (Table).

\section{Timing of Stroke Presentation}

Fifteen children (60\%) had evidence of cerebral infarction on imaging (On-line Table 1). The mean age at which infarction was first identified on neuroimaging was 6.8 years (range, $0.4-10.7$ years). Infarct was noted on the initial imaging study in $11 / 15$ (73\%). In 6/11 (55\%) with infarction noted on the first imaging study, there were no acute neurologic symptoms, suggesting that these were clinically silent infarcts. Stroke incidence in boys was 9/14 (64\%) versus 6/11 (55\%) in girls. The Kaplan-Meier model of stroke-free probability estimated $50 \%$ of children to have radiographically detectable stroke by the age of 8 years (95\% confidence interval, 5.8-10.3 years) (Fig 1). Ten children (40\%) did not demonstrate any infarct on MR imaging during the period of radiologic observation (oldest patient ages at imaging ranged from birth to 12.2 years).

\section{Pattern of Infarction and Vascular Changes}

The spectrum of infarct topography included arterial territorial, white matter, lacunar, and watershed infarcts (Figs 2 and 3). 


\begin{tabular}{lc}
\multicolumn{1}{c}{ Indications $^{\mathbf{a}}$} & $\begin{array}{c}\text { No. of } \\
\text { Scans }\end{array}$ \\
\hline Acute neurologic symptoms suspicious for TIA/stroke & 50 \\
Screening/diagnostic & 31 \\
Follow-up on prior imaging & 21 \\
Seizure & 13 \\
Unknown & 8 \\
Trauma & 7 \\
Headaches & 5 \\
Other & 5 \\
Extra-axial hemorrhage/subdural hematoma & 3 \\
Papilledema & 2 \\
Growth hormone initiation & 1 \\
\hline
\end{tabular}

a Some patients had $>1$ indication for imaging and/or multiple studies done at 1 study date.

${ }^{\mathrm{b}}$ Eye pain, stent placement, cyst evaluation.

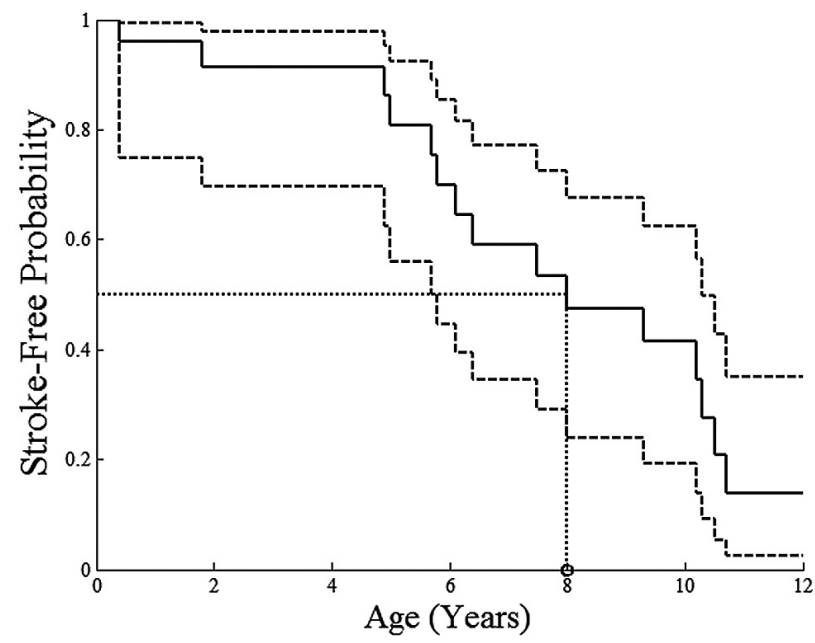

FIG 1. Kaplan-Meier model of stroke-free probability depicts the youngest ages of patients at which imaging demonstrated $\geq 1$ infarct, acute or chronic. The solid line shows the estimated stroke-free probability; dashed lines, the $95 \% \mathrm{Cls}$; and the dotted lines, the estimated age at which $50 \%$ of children have radiographically detectable stroke.

There were 16 large-vessel territorial infarcts, 7 watershed infarcts, 15 focal deep and/or subcortical white matter infarcts, and 5 lacunar infarcts. Nonspecific white matter changes were seen in 11 patients. The large-vessel territorial infarcts were seen in all vascular territories, with a predominance of MCA infarcts. No brainstem, cerebellar, or hemorrhagic infarcts were observed.

On-line Table 2 shows the neurovascular imaging characteristics. Vascular assessment of the neck was available in 10/25 (40\%) patients. We noted narrowing of the cervical CCA in $1 / 10(10 \%)$, narrowing of the cervical ICA in $3 / 10(30 \%)$, and stenosis of the origin of the cervical VA in $1 / 9$ (11\%). Calcification of the CCA, cervical ICA, external carotid artery, or VA was identified in $1 / 2$ (50\%) patients (Fig 4). A robust finding was narrowing of the distal V2/proximal V3 segment of the VA centered at the C2 level, which was seen in $9 / 11(82 \%)$ patients (Fig 5). The presence of prominent collateral vessels in the neck, in conjunction with narrowing of the distal V2/proximal V3 segment of the VA, was evidenced by enlargement of deep cervical arteries in 7/9 (78\%), ascending cervical arteries in 4/10 (40\%), and occipital arteries in $7 / 10(70 \%)$ studies (Fig 5). Enlargement and tortuosity of the

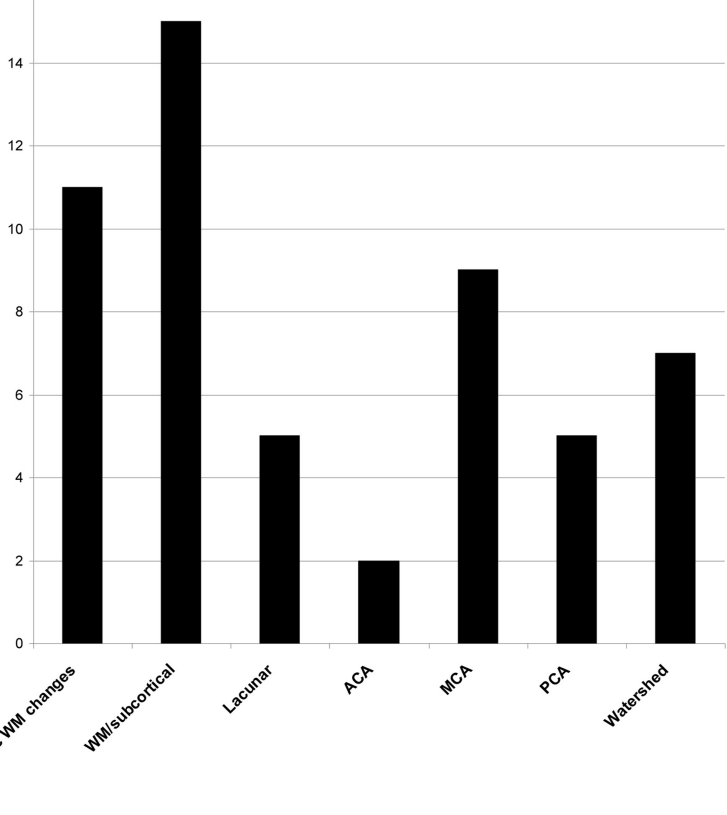

FIG 2. Graphic representation of infarct distribution.

anterior and posterior spinal arteries were seen in 8/10 (80\%) and $3 / 10(30 \%)$ patients, respectively (Fig 6).

The most frequently noted intracranial arterial stenoses involved the ICA (Fig 7). There was narrowing or occlusion of the precavernous ICA in $11 / 13$ (85\%), the cavernous ICA in $12 / 13$ (92\%), and the supraclinoid ICA in 7/13 (54\%) patients, respectively. Narrowing of the ACAs and MCAs was frequently seen in $8 / 13(62 \%)$ and $6 / 13(46 \%)$ patients, respectively, while narrowing of the PCAs was less commonly observed in 2/14 (14\%). No patient demonstrated narrowing of the V4 segment of the VA or narrowing of the basilar artery.

Collateral vessels were a common finding within the suprasellar, subfrontal, and perisplenial regions (Fig 8). Bright signal on FLAIR images within cortical sulci, representing slow cortical collateral flow, was seen ipsilateral to arterial stenoses in 14/21 (67\%) cases (Fig 3B). Enlargement of the internal maxillary arteries in $6 / 11(55 \%)$ studies and middle meningeal arteries in 5/11 (45\%) studies was considered a marker for external carotid branch collateral supply to the brain.

No aneurysm, arterial dissection, AVM, arteriovenous fistula, venous sinus thrombosis, or subarachnoid blood was observed.

\section{DISCUSSION}

\section{Neurovascular Arteriopathy in HGPS}

Vascular abnormalities in the brain are commonly found in children with HGPS. We observed stenosis/occlusions of the cavernous and/or precavernous ICAs in $92 \%$ of patients. Narrowing of the ACA, MCA, and PCA was also observed, though no patient had narrowing or occlusion of the V4 segment of the VA or basilar artery. Prior reports have suggested that these vascular changes in HGPS are a manifestation of atherosclerotic disease. ${ }^{16-19}$ However, although both cerebrovascular atherosclerosis and HGPS demonstrate narrowing and occlusive disease of major intracra- 

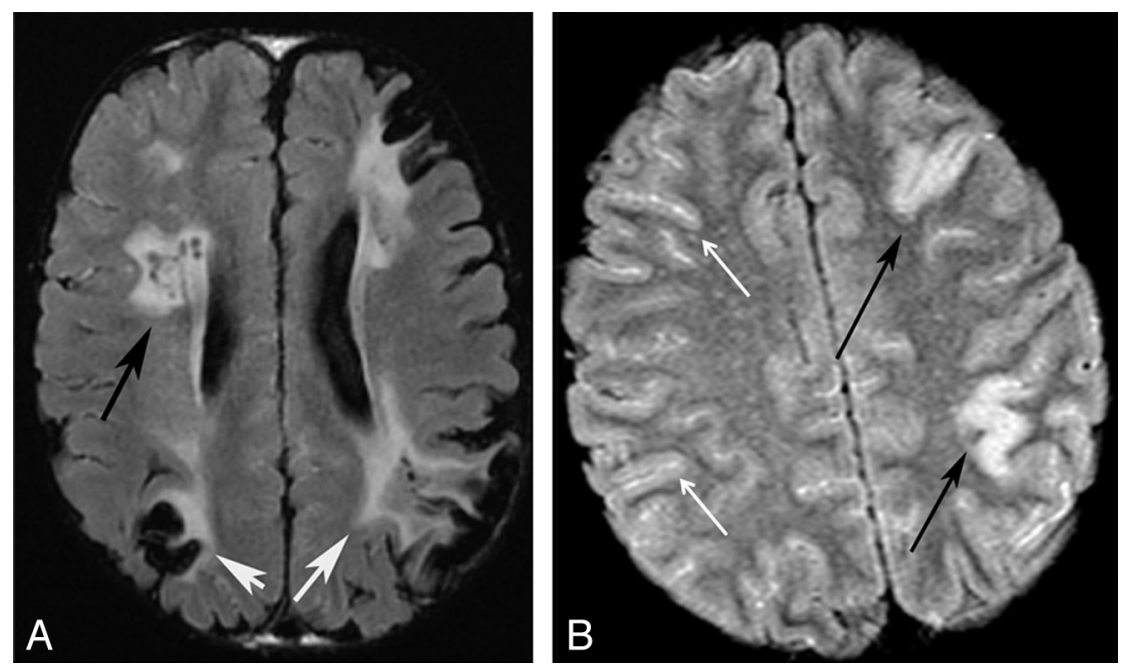

FIG 3. Patterns of infarction. Axial FLAIR images of 2 different patients. A, Chronic watershed (white arrows) and white matter infarcts (black arrow). B, Acute gyral infarcts (black arrows). Bright signal in the sulci indicates slow cortical collateral flow (white arrows).
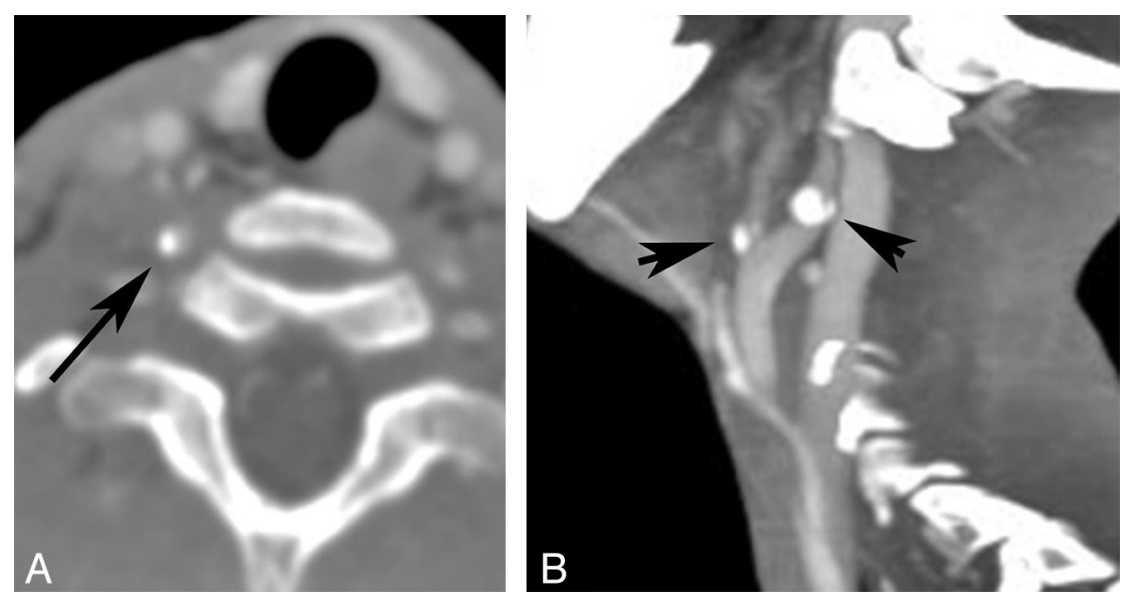

FIG 4. Arterial calcification. CTA reformatted images of the same patient demonstrate right VA calcification $(A)$ and ICA and external carotid artery $(B)$ calcifications.
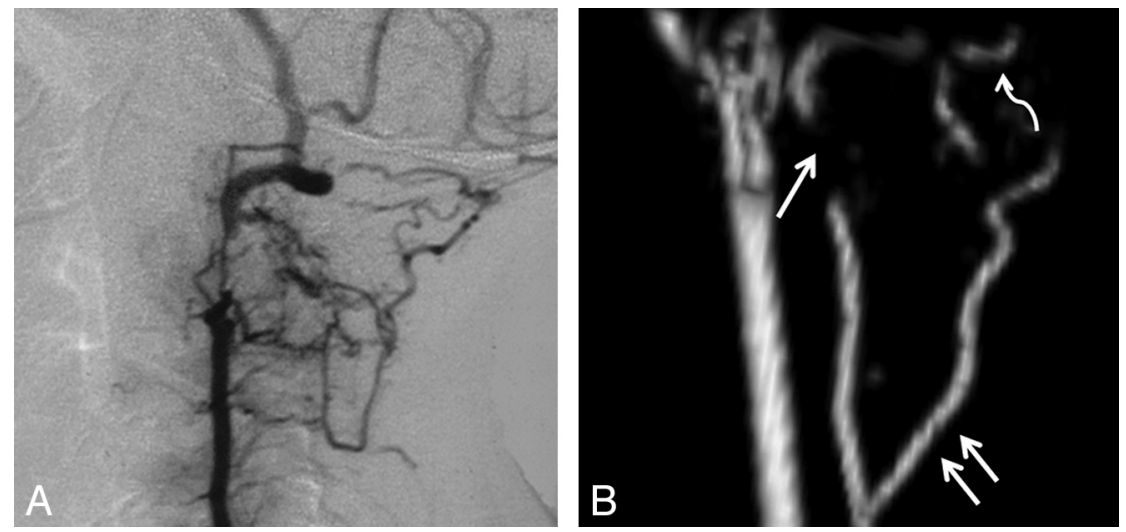

FIG 5. VA stenoses and collaterals. A, Lateral projection of a conventional angiogram demonstrates a short-segment high-grade stenosis of the distal V2 segment of the VA. B, Lateral projection of a 3D model of the cervical MRA in a different patient demonstrates a high-grade stenosis of the V2 segment of the VA (single white arrow), an enlarged deep cervical artery (double white arrows), and enlargement of the occipital artery (curved white arrow). nial arteries, the distribution of neurovascular findings observed in our cohort deviates from what is classically described in intracranial atherosclerosis in the elderly. Intracranial atherosclerosis typically involves the cavernous ICA, MCA, midbasilar artery, V4 segments of the VA, and the PCA. Furthermore, in adult atherosclerosis, MCA narrowing is more common than is ACA stenosis, in contrast to our study population, in which the inverse was observed.

Among the most frequent observations in our cohort were proliferative collateral vessel formation within the suprasellar, subfrontal, and perisplenial regions; enlargement of the internal maxillary and middle meningeal arteries; and slow compensatory collateral convexity flow. These findings do not resemble the known imaging manifestations of atherosclerosis. Thus, we hypothesize that the pattern of the arterial pathology we observed likely represents a steno-occlusive vasculopathy unique to HGPS. This hypothesis is further supported by the frequent and unusual finding of a severe shortsegment distal V2/proximal V3 VA stenosis, which was accompanied by prominent collateral vessel formation involving the ascending, deep cervical, occipital, and anterior and posterior spinal arteries.

Many of the intracranial findings identified in our cohort mimic those found in Moyamoya, a rare progressive occlusive disease of the cerebral vasculature. Moyamoya is characterized by proliferative collateral vessel formation within the basal cisterns, enlargement of the internal maxillary and middle meningeal arteries, and slow collateral convexity flow. However, the pattern and distribution of the intracranial arterial stenoses in HGPS differ from those in Moyamoya in several important ways. Whereas narrowing and occlusion of the precavernous or cavernous segments of the ICA are commonly observed in HGPS, stenoses of the ICA terminus and proximal ACA and MCA are most often seen in Moyamoya. In our study, the ACA was more frequently stenosed than the MCA, which is opposite to what is seen in Moyamoya. Furthermore, Moyamoya does not typically involve the cervical vasculature or the distal V2/proximal V3 segment of the VA and does not involve enlargement of the anterior or posterior spinal arteries. 

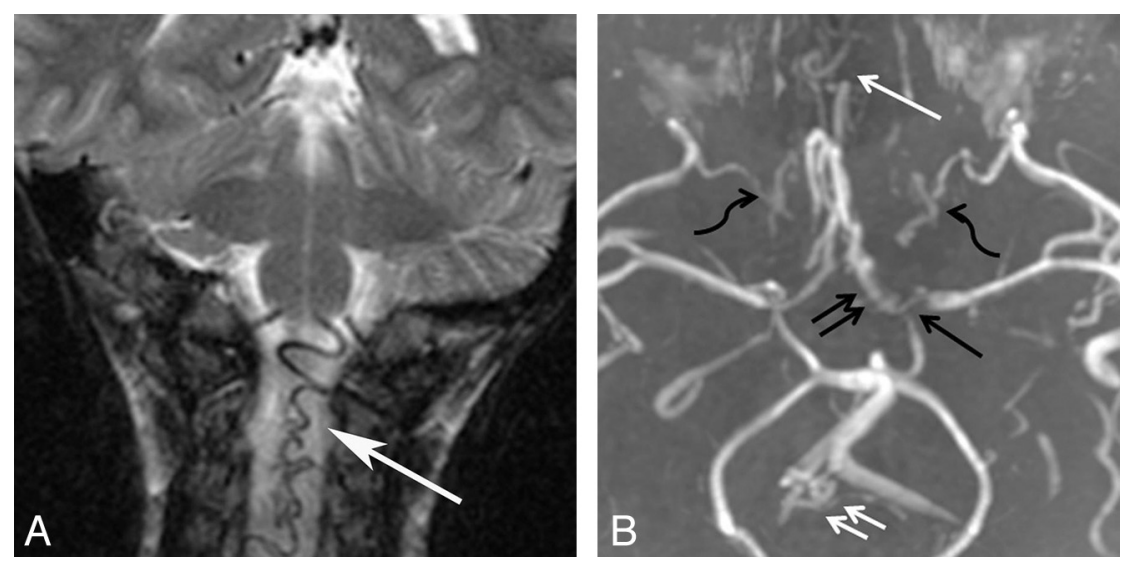

the anterior spinal artery is typically associated with spinal AVMs or arteriovenous fistulas.

The location of disease in HGPS most commonly involves a short segment of the cavernous or precavernous ICA and a distal segment of the cervical VA, in addition to the proximal ACA and MCA. This uniform pattern of disease may reflect a segmental vulnerability of the vascular tree, based on arterial embryogenesis and modulated by genetics, to secondary triggers, ${ }^{23}$ such as hypertension, hypercholesterolemia, hyperinsulinemia, and insu-

FIG 6. Collateral vessels. A, Coronal T2WI of an enlarged ASA (white arrow). B, Collapsed view of an MRA in a different patient demonstrates M1 (single black arrow) and Al stenoses (black double arrows), internal maxillary artery collaterals (black wavy arrows), subfrontal collaterals (single white arrow), and an enlarged ASA and posterior spinal artery (white double arrows).

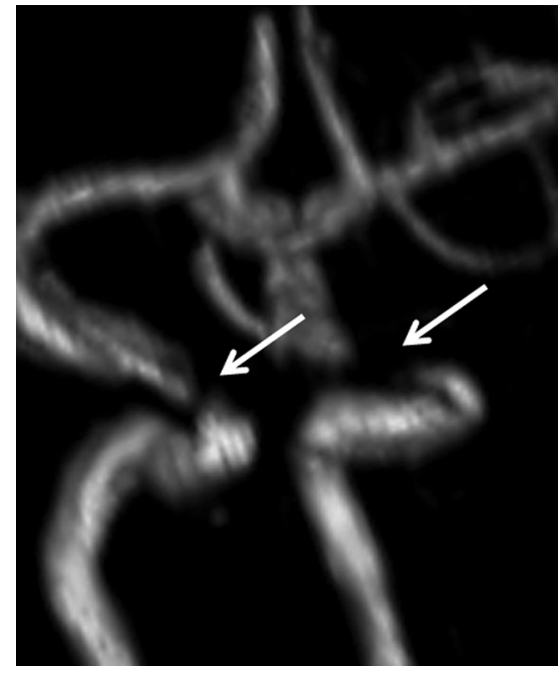

FIG 7. ICA stenosis. 3D model of the MRA of the circle of Willis demonstrates bilateral short-segment high-grade stenoses of the cavernous ICAs (white arrows).

Lenticulostriate proliferation arising from the M1 segment of the MCA is considered highly suggestive of Moyamoya, and yet it was a consistent finding in HGPS. Basal collateral vessels are not considered a feature of intracranial atherosclerosis or other arteriopathies such as intracranial arterial dissection, focal cerebral arteriopathy of childhood, or CNS vasculitis. The high prevalence of distal V2/proximal V3 segment VA stenosis, associated with enlargement of the anterior spinal artery, is also notable. The enlargement of the anterior spinal artery most likely represents an unusual arterial collateral pathway, which, in conjunction with enlarged occipital, ascending, and deep cervical arteries, serves to bypass vertebral artery stenoses, when present. Enlargement of the anterior spinal artery is rarely seen in the adult population and is described as a rare collateral pathway in patients with severe bilateral atherosclerotic disease involving the V4 segments of the VA. ${ }^{20-22}$ Enlargement of the anterior spinal artery has previously been described in a single case of a 4-year-old child with HGPS and bilateral VA-origin occlusions. ${ }^{19}$ In children, enlargement of rysm was identified in our cohort, nor were brain vascular malformations, such as AVMs or dural or pial arteriovenous fistulas, seen as can be observed in children with RASA1 mutations and hereditary hemorrhagic telangiectasia. Furthermore, the absence of imaging evidence for arterial dissection, vascular ectasia, or fusiform arteriopathy strongly suggests that the vascular pathology in HGPS differs from that of connective tissue disorders such as Loeys-Dietz and Ehlers-Danlos syndromes.

In our assessment of the vascular pathology of the mid and inferior neck, we were limited by the lack of dedicated neck imaging studies. However, we were able to identify CCA, ICA, and VA narrowing and calcification, with appearances similar to those seen in cervical arterial disease in adult atherosclerosis. In addition, arterial plaque has previously been identified within the carotid arteries by sonography in children with HGPS. ${ }^{9,10}$ These findings suggest that the arteries within the mid/lower neck in HGPS have more in common with the atherosclerotic-like pathology of the coronary arteries seen in HGPS than they do with the steno-occlusive lesions in the brain.

In our cohort, the severity of the intracranial arterial disease was disproportionate and more severe than the disease within the neck. This is opposite to what is observed in adult atherosclerosis in which cervical occlusive disease predominates. This disparity also suggests that the arterial disease within the neck may differ from the arteriopathy within the brain. The differences in the manifestation of arterial disease in HGPS may be explained by differences between the intracranial and peripheral vasculature in general. Intracranial arteries lack a vascular adventitia and have a different sympathetic vascular innervation and responsivity to systemic hypertension than arteries in the neck. Targeted postmortem studies are needed for further evaluation.

\section{Stroke in HGPS}

Arterial ischemic stroke, with or without clinical symptoms, was seen in $60 \%$ of our patients. In patients with reports of focal neurologic deficits, symptoms were similar to those typically found in children with stroke secondary to other etiologies such as embolism, dissection, and focal cerebral arteriopathy of childhood. ${ }^{24}$ In $44 \%$ of patients, infarct was noted on the first imaging study. Most 

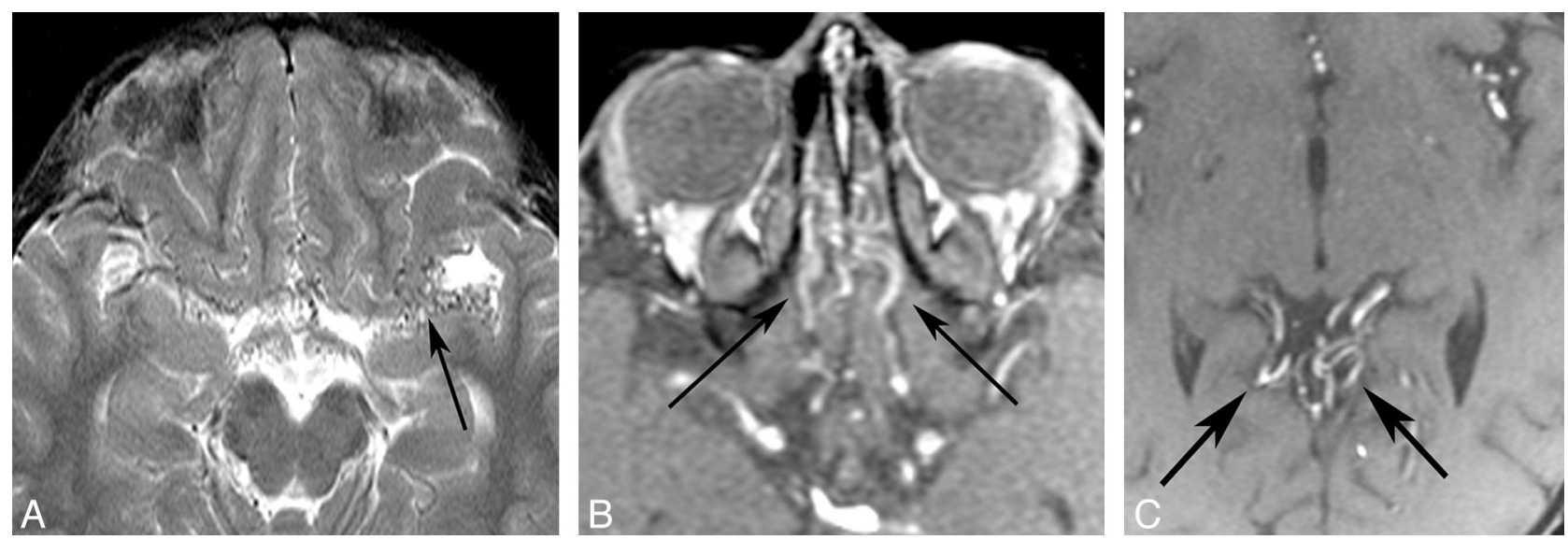

FIG 8. Collateral vessels. A, Axial T2WI shows collateral vessels in the basal cistern (arrow). Source images from an MRA in a different patient demonstrate subfrontal $(B)$ and perisplenial $(C)$ collateral vessels (arrows.)

important, no preceding stroke-like symptoms were present in more than half of these patients, suggesting that many infarcts in children with HGPS are clinically silent. Most infarcts identified on the first available imaging were in patients between 5 and 10 years of age, suggesting that stroke onset does not cluster toward the end of life when TIAs and clinically evident strokes are a more frequent cause of morbidity and mortality.

Stroke distribution included large-vessel territorial, white matter, watershed, and lacunar infarcts. The large-vessel infarctions involved all vascular territories, with a predilection for the MCA and watershed regions. PCA infarcts were observed despite the rarity of PCA stenosis. No brain stem or cerebellar infarcts were seen, despite the high prevalence of distal V2/proximal V3 segment VA stenosis, which suggests that the collateral support derived from the spinal, cervical, and occipital arteries was adequate.

Confluent white matter changes within the cerebral hemispheres were commonly seen; these may represent ischemic injury of cerebral white matter secondary to chronic hypoperfusion. ${ }^{25}$

The absence of hemorrhagic stroke was notable, given that hemorrhagic stroke is estimated to account for approximately half of all childhood stroke with hematologic abnormalities reported as the major risk factor in most series. ${ }^{26}$ Similar to HGPS, children with Moyamoya also most commonly experience ischemic stroke. $^{27}$

It has not yet been established whether stroke in HGPS results from the progressive cerebral arteriopathy, narrowing and/or occlusion of the arteries within the neck, emboli arising from atheromatous plaque within arteries of the neck, emboli arising from a cardiac source, or a combination of the aforementioned processes. Hypercoagulopathy is not a feature of HGPS and thus an unlikely cause of stroke in this population. ${ }^{9}$

\section{Imaging Recommendations}

Given our current understanding of HGPS, we recommend a baseline MRI of the brain and MRA of the head and neck to determine the presence and severity of cervical and intracranial disease and frequent clinical monitoring for arterial ischemic stroke.

\section{CONCLUSIONS}

We present the imaging findings related to cerebrovascular disease in the only existing neurovascular cohort study of HGPS. Arterial ischemic stroke in HGPS is common and often clinically silent. The findings of intracranial and distal VA steno-occlusive disease with collateral vessel formation and slow collateral convexity flow suggest an intracranial vasculopathy unique to HGPS; these findings are distinct from other vasculopathies of childhood and from intracranial atherosclerotic disease in the aged. Furthermore, while children with HGPS share some imaging features with Moyamoya, the location of stenoses differs; therefore, HGPS is not merely a variant of Moyamoya. The vascular imaging characteristics of the mid/inferior neck are less specific and share more similarities with atherosclerotic-like disease of the coronary arteries, also identified in HGPS, than they do with steno-occlusive lesions in the brain. This study was inherently limited by the retrospective nature of this heterogeneous cohort with variability in terms of the number and quality of imaging studies. Definitive characterization of the cerebrovascular pathology will require pathologic examination of the vessels of the head and neck. Targeted interventions for HGPS that include neuroimaging evaluations as part of a secondary outcome are being developed. It will be essential to determine whether such interventions slow the progression of vasculopathy and, ultimately, result in improved neurologic outcomes in children with HGPS.

\section{ACKNOWLEDGMENTS}

We are grateful to the families and children in our cohort for study participation. We thank Nancy Wolf-Jensen and Joan Brazier for data base assistance and Nancy Drinan for manuscript preparation.

Disclosures: Leslie Gordon—RELATED: Grant: The Progeria Research Foundation, * Comments: grant to Rhode Island Hospital to support study activities, UNRELATED: Employment: Rhode Island Hospital,* Comments: I am employed by Rhode Island Hospital and receive grants, which include salary, from this employment, Grants/ Grants Pending: The Progeria Research Foundation (nonprofit 5013C), ${ }^{*}$ National Institutes of Health, ${ }^{*}$ Comments: multiple grants. Each is an academic award for research conducted at Rhode Island Hospital, Brown University, or Boston Children's Hospital. Each award is associated with an institutional review board-approved project, Payment for Lectures (including service on Speakers Bureaus): I have received honorarium for speaking at academic meetings. All are under $\$ 1000$, Patents 
(planned, pending, or issued): I have several patents, but they are not patents related to receiving money but are related to Progeria syndrome, Travel/Accommodations/Meeting Expenses Unrelated to Activities Listed: The Progeria Research Foundation; OTHER RELATIONSHIPS: I am the parent of a child whose data are part of this study. There was no financial payment for that participation. I am the Medical Director for The Progeria Research Foundation. I do not receive payment for this activity, and I am not a member of the Board of Directors for this nonprofit institution. *Money paid to the institution.

\section{REFERENCES}

1. Hennekam RC. Hutchinson-Gilford progeria syndrome: review of the phenotype. Am J Med Genet A 2006;140:2603-24

2. Eriksson M, Brown WT, Gordon LB, et al. Recurrent de novo point mutations in lamin A cause Hutchinson-Gilford progeria syndrome. Nature 2003;423:293-98

3. De Sandre-Giovannoli A, Bernard R, Cau P, et al. Lamin A truncation in Hutchinson-Gilford progeria. Science 2003;300:2055

4. Goldman RD, Shumaker DK, Erdos MR, et al. Accumulation of mutant lamin A causes progressive changes in nuclear architecture in Hutchinson-Gilford progeria syndrome. Proc Natl Acad Sci U S A 2004;101:8963-68

5. Varga R, Eriksson M, Erdos MR, et al. Progressive vascular smooth muscle cell defects in a mouse model of Hutchinson-Gilford progeria syndrome. Proc Natl Acad Sci U S A 2006;103:3250-55

6. Olive M, Harten I, Mitchell R, et al. Cardiovascular pathology in Hutchinson-Gilford progeria: correlation with the vascular pathology of aging. Arterioscler Thromb Vasc Biol 2010;30:2301-09

7. Conneely KN, Capell BC, Erdos MR, et al. Human longevity and common variations in the LMNA gene: a meta-analysis. Aging Cell 2012;11:475-81

8. Kieran MW, Gordon L, Kleinman M. New approaches to progeria. Pediatrics 2007;120:834-41

9. Merideth MA, Gordon LB, Clauss S, et al. Phenotype and course of Hutchinson-Gilford progeria syndrome. N Engl J Med 2008;358: 592-604

10. Gerhard-Herman M, Smoot LB, Wake N, et al. Mechanisms of premature vascular aging in children with Hutchinson-Gilford progeria syndrome. Hypertension 2012;59:92-97

11. Makous N, Friedman S, Yakovac W, et al. Cardiovascular manifestations in progeria: report of clinical and pathologic findings in a patient with severe arteriosclerotic heart disease and aortic stenosis. Am Heart J 1962;64:334-46

12. Baker PB, Baba N, Boesel CP. Cardiovascular abnormalities in progeria. Case report and review of the literature. Arch Pathol Lab Med 1981;105:384-86

13. Atkins L. Progeria: report of a case with post-mortem findings. N Engl J Med 1954;250:1065-69

14. Ullrich NJ, Silvera VM, Campbell SE, et al. Craniofacial abnormalities in Hutchinson-Gilford progeria syndrome. AJNR Am J Neuroradiol 2012;33:1512-18

15. Moulson CL, Fong LG, Gardner JM, et al. Increased progerin expression associated with unusual LMNA mutations causes severe progeroid syndromes. Hum Mutat 2007;28:882-89

16. Wagle WA, Haller JS, Cousins JP. Cerebral infarction in progeria Pediatr Neurol 1992;8:476-77

17. Rosman NP, Anselm I, Bhadelia RA. Progressive intracranial vascular disease with strokes and seizures in a boy with progeria. $J$ Child Neurol 2001;16:212-15

18. Alghamdi SA. A case of progeria in a Saudi child presenting with cerebral infarction. Ann Saudi Med 1995;15:631-33

19. Smith AS, Wiznitzer M, Karaman BA, et al. MRA detection of vascular occlusion in a child with progeria. AJNR Am J Neuroradiol 1993; 14:441-43

20. Kang HS, Han MH, Kim SH, et al. Anterior spinal artery as a collateral channel in cases of bilateral vertebral arterial steno-occlusive diseases. AJNR Am J Neuroradiol 2007;28:222-25

21. Hott JS, Vishteh G, Wallace R, et al. Anterior spinal artery supplying posterior circulation. Neurology 2004;62:468

22. Garnier P, Januel AC, Demasles S, et al. Collateralization of vertebral arteries. Neurology 2003;60:720

23. Lasjaunias PL. Segmental identity and vulnerability in cerebral arteries. Interv Neuroradiol 2000;6:113-24

24. Tsze DS, Valente JH. Pediatric stroke: a review. Emerg Med Int 2011; 2011:734506

25. Pantoni L, Garcia JH. Pathogenesis of leukoaraiosis: a review. Stroke 1997;28:652-59

26. Jordan LC, Hillis AE. Hemorrhagic stroke in children. Pediatr Neurol 2007;36:73-80

27. Scott RM, Smith ER. Moyamoya disease and Moyamoya syndrome. N Engl J Med 2009;360:1226-37 